\title{
Tunable Raman fiber laser induced by Rayleigh back-scattering in an ultra-long cavity
}

A. R. Sarmani

\section{R. Zamiri}

\section{H. Abu Bakar}

B. Z. Azmi

\section{A. W. Zaidan}

M. A. Mahdi

mdadzir@eng.upm.edu.my
Wireless and Photonics Network Research Center, Faculty of Engineering, University Putra Malaysia, 43400 UPM Serdang, Selangor, Malaysia

Department of Physics, Faculty of Science, Universiti Putra Malaysia, 43400 UPM Serdang, Selangor, Malaysia

Wireless and Photonics Network Research Center, Faculty of Engineering, University Putra Malaysia, 43400 UPM Serdang, Selangor, Malaysia

Department of Physics, Faculty of Science, Universiti Putra Malaysia, 43400 UPM Serdang, Selangor, Malaysia

Department of Physics, Faculty of Science, Universiti Putra Malaysia, 43400 UPM Serdang, Selangor, Malaysia

Wireless and Photonics Network Research Center, Faculty of Engineering, University Putra Malaysia, 43400 UPM Serdang, Selangor, Malaysia

We report an ultra-long Raman laser with a $46 \mathrm{~km}$ fiber length that behaved as a Rayleigh back-scattering-based optical feedback. The laser was tunable from $1550 \mathrm{~nm}$ to $1571 \mathrm{~nm}$ (3 dB bandwidth) with the insertion of an optical grating filter. Evaluations on the spectral evolution and power development were also performed from the results obtained. In fact, it was discovered that the spectral broadening effect between the modeless spectra resembled the same process that happens in a typical fiber cavity that has high reflectors at each cavity end. In addition, the output power showed a square-root development with respect to the input power. [DOI: http://dx.doi.org/10.2971/jeos.2011.11043]

Keywords: Raman fiber laser, Rayleigh scattering, spectral broadening

\section{INTRODUCTION}

Raman fiber lasers (RFLs) are favorable coherent light sources that can be designed to operate in the near infrared region which can cover from $1.1 \mu \mathrm{m}$ to $1.6 \mu \mathrm{m}$ wavelengths. A careful selection of pump wavelengths and appropriate spectroscopic properties of the gain medium are the main criteria that determine this wide range of tunability. Together with other fascinating technical attributes such as wide amplification bandwidth and tailored to any wavelength range, RFLs have been utilized to generate multiple wavelength lasers [1]. Other beneficial applications are for optical coherence tomography, pulse compression, supercontinuum generation, and spectroscopy [2].

Lasing in a Raman fiber resonator [3] involves the exploitation of a nonlinear optical effect that is known as stimulated Raman scattering (SRS). In SRS, an inelastic process occurs where the initial frequency of an electromagnetic radiation that circulates in an optical fiber is downshifted towards lower Stokes frequency components. These were firstly demonstrated in 1988 by incorporating fiber Bragg gratings as cavity reflectors instead of conventional mirrors [4]. A strong SRS influence can be produced in fiber waveguides due to their tiny core diameters around 5-10 $\mu \mathrm{m}$ and low absorption property, $\alpha \sim 0.2 \mathrm{~dB} / \mathrm{km}$ at $1550 \mathrm{~nm}$. These allow a high- intensity intracavity light propagation that inspires the development of ultralong Raman fiber lasers (URFLs) for quasi-lossless communication links [5]. In these laser schemes that consisted of a visible optical cavity, the resolvable mode structure could still be observed even though fiber lengths up to $270 \mathrm{~km} \mathrm{[6]} \mathrm{were}$ utilized.

The operation of URFLs is strongly related to Rayleigh backscattering (RBS) where the frequency of scattered light remains unchanged. RBS that is an example of elastic scattering is a critical source of fiber losses. It is induced by microscopic nonuniformities of refractive index in the gain medium in which light propagates. Although the influence of RBS in a typical fiber that has a high-Q cavity is negligible at $1550 \mathrm{~nm}$ wavelength, it might be important in an extreme cavity length dimension $(\sim 100 \mathrm{~km})$. In a few research papers, it has been discovered that tens of $\mathrm{km}$ fiber length could behave as a virtually distributed mirror to produce Raman lasing [7, 8]. In these asymmetric configurations that consisted of an FBG or a fiber loop mirror at one side of the cavity, the optical feedback at another end was instigated by the RBS mechanism. No formation of a standing wave cavity was reported in this modeless laser spectrum as verified in [9]. However at the present time, no sufficient theoretical and numerical models have been developed to increase our understanding with regards to this new physical effect. Therefore in this letter, we 
provide an experimental report that clarifies the contribution of RBS effect to the lasing properties, namely tuning ranges, power development, and spectral evolutions.

\section{EXPERIMENTAL SETUP}

The widely tunable URFL was designed in a configuration that included a Raman pump unit (RPU) at $1455 \mathrm{~nm}$ and an optical grating filter (OGF) as illustrated in Figure 1. The pump source that had a maximum output power of $1500 \mathrm{~mW}$ was spliced to the fiber system via a $1480 / 1550 \mathrm{~nm}$ wavelength selective coupler (WSC). The OGF utilized in this architecture is tunable from $1525 \mathrm{~nm}$ to $1625 \mathrm{~nm}$ with a $-3 \mathrm{~dB}$ bandwidth of $0.22 \mathrm{~nm}, \mathrm{a}-20 \mathrm{~dB}$ bandwidth of $0.60 \mathrm{~nm}$ and an insertion loss of $7 \mathrm{~dB}$. Both its ends were connected to an optical circulator (OC)-based loop mirror. In the previous assessments, the lasing feedback in the fiber system was provided by highly reflective fiber Bragg gratings (FBG) [3, 4]. However, in this laser scheme the optical cavity was completed by a virtually distributed mirror that implies Rayleigh backscattering impact [7, 8]. This was realized by using a $46 \mathrm{~km}$ length of single-mode fiber of type SMF-28 which has a core diameter of $8.2 \mu \mathrm{m}$ and numerical aperture of 0.14 . At $1550 \mathrm{~nm}$, the attenuation and mode-field diameter of the fiber is $0.22 \mathrm{~dB} / \mathrm{km}$ and $10.4 \pm 0.8 \mu \mathrm{m}$ respectively while the dispersion is $17 \mathrm{ps} /(\mathrm{nm} . \mathrm{km})$ with a zero dispersion slope of $0.086 \mathrm{ps} /\left(\mathrm{nm}^{2} . \mathrm{km}\right)$. The fiber is coupled to an isolator to prevent Fresnel reflection from the fiber end as it permits the propagation of Raman and remaining pump photons in only one direction. This ensured that only the returning photons that were contributed by Rayleigh backscattering effect (RBS) could bounce back and forth in the fiber system.

When the required threshold for Raman gain to overcome the fiber attenuation and losses caused by the absence of lumped mirrors at one end of the cavity was achieved, lasing was initiated. This was detected at the $1 \%$ output port that was provided by a 99:1 splitter which was located in between the WSC and the gain medium. The resulting first Stokes wavelength was expected to be around $1555 \mathrm{~nm}$ that corresponds to the $13 \mathrm{THz}$ SRS shift in the silica. The spectral profiles were measured by utilizing an optical spectrum analyzer (OSA) with a resolution of $0.05 \mathrm{~nm}$.

\section{RESULTS AND DISCUSSION}

At the outset of these evaluations, the input power from RPU unit was intensified gradually to $1500 \mathrm{~mW}$ with $50 \mathrm{~mW}$ increments. At each power value, the OGF was tuned within its accessible wavelength range and the spectral features obtained on the OSA were analyzed individually. In this case, the existence of an optical spectrum that has exponential-wing envelopes and a narrow peak that corresponds to its central wavelength represented a lasing condition. The peak amplitude for each laser signal was then recorded and the tunability data was plotted as a function of wavelengths as manifested inFigure 2(a). The measured lasing range overlapped agreeably with the bandwidth of SRS which was around $105 \mathrm{~nm}$ away from its pump wavelength at $1455 \mathrm{~nm}$. From 3-dB band-

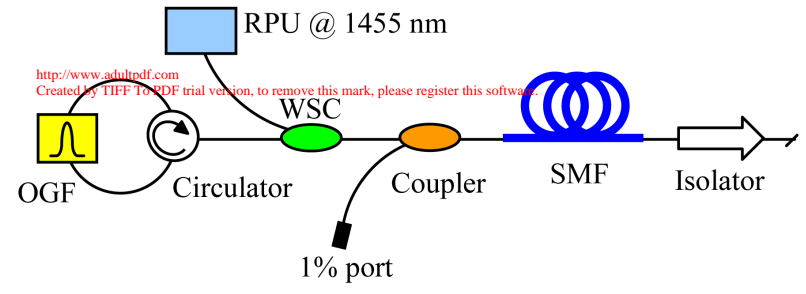

FIG. 1 Experimental set-up of an ultra-long Raman fiber laser.
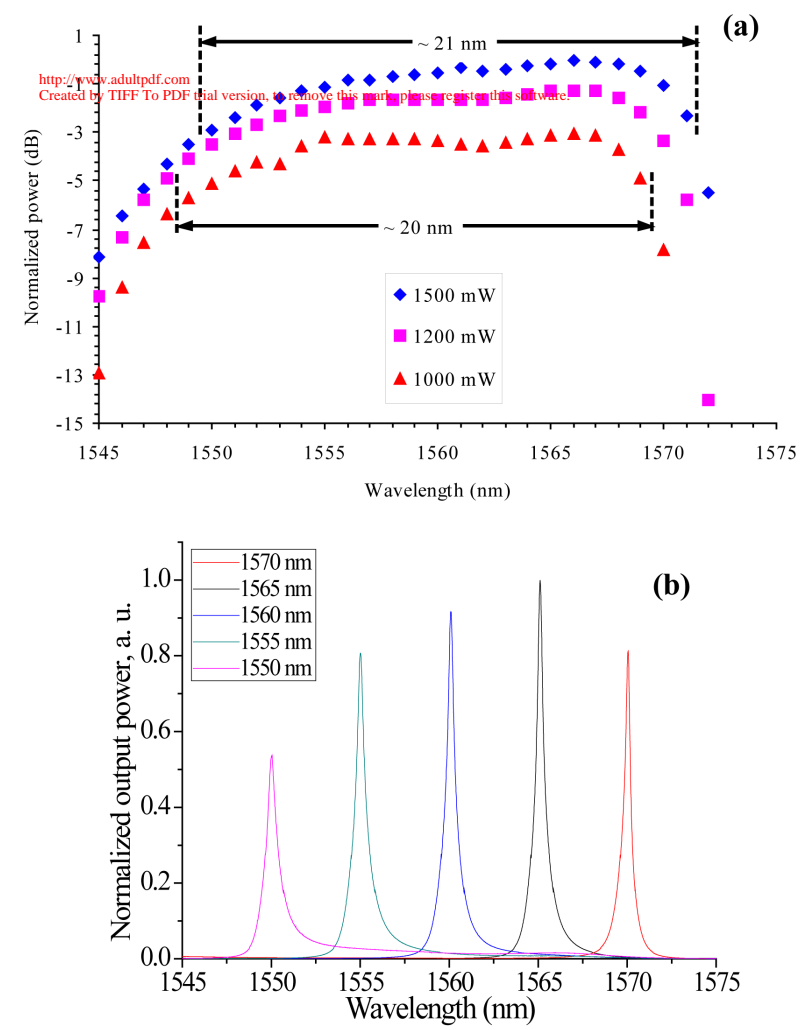

FIC. 2 (a) Measured output power of laser at $1 \%$ port of coupler and (b) laser spectra at a maximum pump power of $1500 \mathrm{~mW}$.

width of the graph, the tuning was in the vicinity of $20 \mathrm{~nm}$ $(1549-1569 \mathrm{~nm})$ at a pump power of $1000 \mathrm{~mW}$. It then expanded slightly to $21 \mathrm{~nm}(1550-1571 \mathrm{~nm})$ when the pump power reached its maximum value at $1500 \mathrm{~mW}$. An example of optical spectra obtained at this power level is presented in Figure 2(b). From this figure, the laser spectra were measured without any ripples at all wavelength regions.

An almost similar configuration to this setup was also constructed by Babin et. al. with a fiber length of $41 \mathrm{~km}$ [8]. It was found that the intracavity generated radiation in this system has no mode structures that relate to the cavity round trip time, $\tau_{r t}=2 \mathrm{~nL} / \mathrm{c}$. This physical issue can be explained microscopically. With the exclusion of an FBG at one side of the cavity, the long sectioned-fiber span behaves as a distributed mirror at another end. As the consequence, the Raman photons that propagate earlier in the forward direction are reflected stochastically by RBS effects. These introduce numerous effective intra-cavity resonators with randomly variable lengths from each reflection location. By combining together with Raman photons that propagate in the backward direction, these interacting waves result in a multitude of modes overlapping 
that produces modeless stochastic spectra. Recently, a published paper has elucidated the concept of random lasers in a mirror-less RFL cavity that incorporated an $83 \mathrm{~km}$ fiber [9]. The pump sources were two-equal power of $1455 \mathrm{~nm}$ lasers that were arranged in the transverse direction.

On the other hand, in a conventional fiber laser architecture that comprises a visible optical cavity, the broadening of spectral bandwidth happens as the consequence of nonlinear interactions between the modes. The quasi-degenerate four-wave mixing process that involves a huge number of intracavity Stokes wave longitudinal modes leads to random evolutions of the amplitude and phase of each spectral element. Mathematical explanations based on wave kinetic equations to describe this phenomenon have been reported in [10]. Interestingly in this modeless laser system, the same broadening scheme also occurred for the spectra at $1555 \mathrm{~nm}$ as presented in Figure 3(a). However other contributions of Kerr-lens nonlinearities to this process, especially self-phase or cross-phase modulation (SPM, XPM) are not possible. This is because the random phase shifts implied in this lasing property cannot be initiated by the purely dynamic nature of the SPM and XPM. Both of these modulations alter the mode phases synchronously [11]. As a result, this cancels out the respective parameters included in the wave kinetic equation that was explained in [10]. Thus, we believe that this process is associated to nonlinear responses (multiple four-wave mixing processes) between the interacting modes although the laser demonstrated no mode beatings. To date, to the extent of our knowledge no numerical modeling has been performed before to address the turbulence-induced broadening within this modeless radiations.

In the next assessment, maximum output powers determined from the top of each laser spectra were evaluated with respect to the incoming pump powers. Unlike the prior result (see Figure 2(a) in [10]), the growth of laser output power $P_{\text {out }}$ at $1555 \mathrm{~nm}$ wavelength was in agreement to the square-root law as clearly shown inFigure 3(b), which also reveals initial lasing occurring from pump power of $700 \mathrm{~mW}$. The slope efficiency of the proposed laser was found around $0.125 \%$. This is owing to the RBS effect that has low reflectivity and the output coupling of $1 \%$ from the laser cavity. When deducing the full width at half maximum from Figure 3(a), it was found that the spectral width grew quadratically with the increase in intracavity Stokes wave powers. This is depicted by the red line fitting in Figure 3(c). A maximum spectral width of less than $0.7 \mathrm{~nm}$ was attained for an intracavity power of only $0.85 \mathrm{~mW}$. In contrast, for the comparable fiber length in (Figure 3(a) [11]) the spectral width was two times narrower for the intracavity power of $200 \mathrm{~mW}$ approximately. The discrepancies (power and spectral width characteristics) in comparison to those presented previously $[10,11]$ were due to the different arrangement in the laser design. The laser cavity in $[10,11]$ was designed by integrating high reflectivity FBGs at both ends thus allowing a more efficient light oscillation compared to our setup that utilized Rayleigh scattering. This translates to lower cavity loss and consequently higher Stokes power. Additionally, the high reflectivity of the FBGs is only at a certain wavelength, in contrast to distributed virtual mirror that lacks specific wavelength reflectivity. This means that
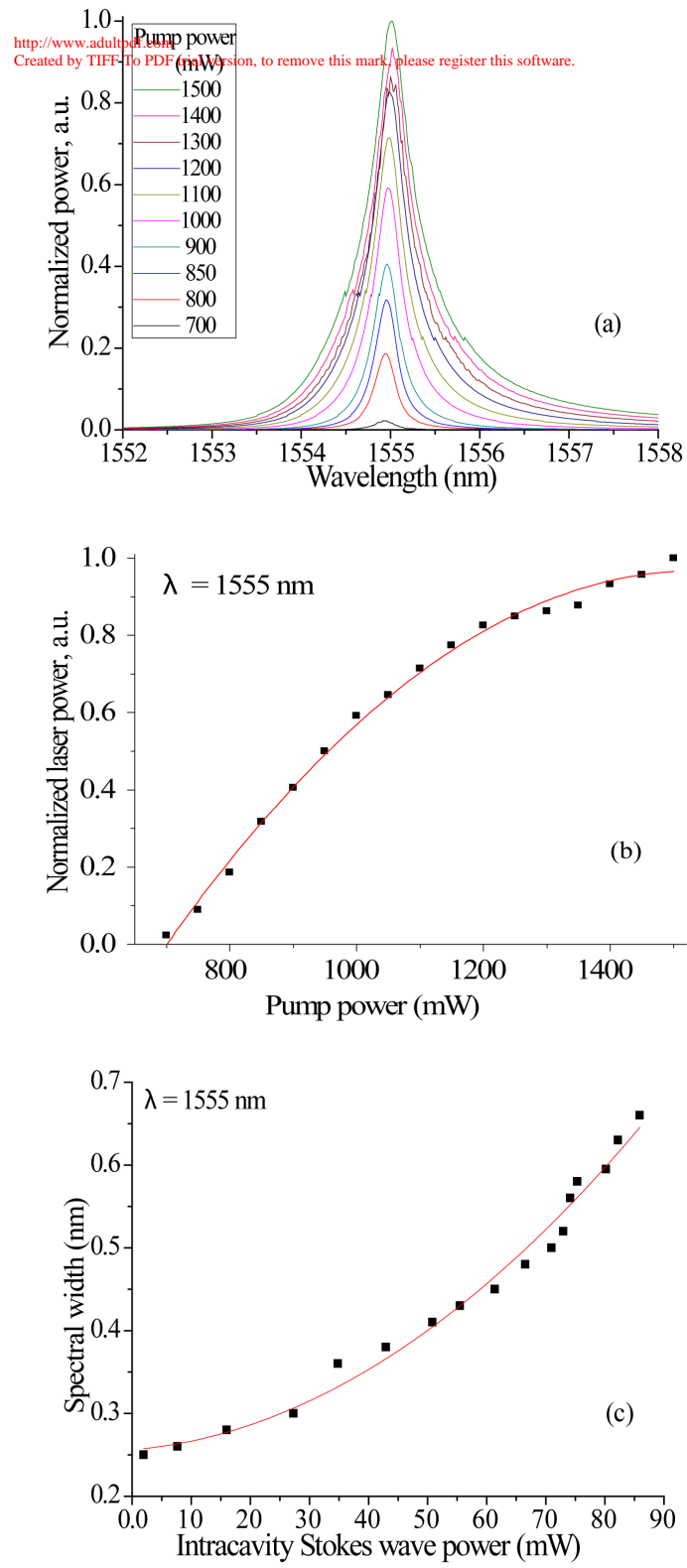

FIG. 3 Lasing performance at $1555 \mathrm{~nm}$, (a) spectral evolutions, (b) their output power characteristics, and (c) is the corresponding spectral width as a function of intracavity powers. The red lines indicate fitting curves of the graph.

random competing modes are reflected back into the cavity, causing the expansion of the spectral wings. As a result, wider spectral width was observed in this experiment even though the Stokes power was lower than in $[10,11]$.

With the incorporation of higher pump powers $\left(P_{p}>1.5 \mathrm{~W}\right)$, the contribution of RBS effect to the laser qualities is expected to be more notable compared to those discussed in this literature. This can further be supported by also including longer fibers as the gain media ( $L>46 \mathrm{~km}$ ). Based on the work reported in [6], in the future we suggest carrying out this research work by employing fiber lengths of more than $200 \mathrm{~km}$. In summary, the development of this type of novel light has an implication of introducing a more simplified configuration that can lead to cost reduction. Besides, some of its equivalent lasing properties compared to those in ordinary fiber lasers 
can open up similar applications in telecommunications, sensing, nonlinear optics and other new emerging areas.

\section{CONCLUSION}

The success of this experiment thus ascertains the role of $46 \mathrm{~km}$ Raman fiber as a RBS-based distributed mirror to complete the laser resonator. With the inclusion of an optical grating filter, this laser had wide tunabilities from $1550 \mathrm{~nm}$ to $1571 \mathrm{~nm}$. This was realized for the pump power of only $1500 \mathrm{~mW}$. The turbulence-induced spectral broadening between modeless laser spectra in this system also occurred in a full analogy to that in a fiber cavity that incorporated two lumped mirrors at each end. Further improvement to these results can be done by using longer fibers with more powerful pump powers to provide more analytical insight about the influence of Rayleigh back-scattering to the lasing parameters. With better understanding of this physical scheme, more advanced design and performance to this new class of lasers can be constructed in the future.

\section{ACKN OWLED GEMENTS}

This work is partly supported by the Ministry of Higher Education, Malaysia and the Universiti Putra Malaysia under post-doctoral research fellowship scheme.

\section{References}

[1] G. Ning, P. Shum, S. Aditya, D. Liu, Y. Gong, N. Q. Ngo, M. Tang "Multiwavelength Raman fiber lasers with equalized peak power using a sampled chirped fiber Bragg grating" Appl. Phys. B 83, 249-253 (2006).

[2] S. Xiao, N. A. Mortensen, and A. P. Jauho "Nanostructure design for surface-enhanced Raman spectroscopy -prospects and limits" J. Eur. Opt. Soc.-Rapid. 3, 08022 (2008).

[3] J. Zhou, J. Chen, X. Li, G. Wu, and Y Wang "Exact analytical solution for Raman fiber laser" IEEE. Photonic. Tech. L. 18, 1097-1099 (2006).

[4] P. N. Kean, B. D. Sinclair, K. Smith, W. Sibbett, C. J. Rowe, and D. C. J. Reid "Experimental evaluation of a fiber Raman oscillator having fiber grating reflectors" J. Mod. Opt. 35, 397-406 (1988).

[5] T. J. Ellingham, J. D. Ania Castanon, R. Ibbotson, X. Chen, L. Zhang, and S. K. Turitsyn "Quasi-lossless optical links for broadband transmission and data processing" IEEE. Photonic. Tech. L. 18, 268270 (2006).

[6] S. K. Turitsyn, J. D. Ania Castanon, S. A. Babin, V. Karalekas, P. Harper, D. V. Churkin, S. I. Kablukov, A. E. El-Taher, E. V. Podivilov, and V. K. Mezentsev "270-km ultralong Raman fiber laser" Phys. Rev. Lett. 103, 133901 (2009).

[7] G. Ravet, A. A. Fotiadi, M. Blondel, and P. Mégret "Passive Qswitching in all-fiber Raman laser with distributed Rayleigh feedback" Electron. Lett. 40, 528-529 (2004).

[8] S. A. Babin, A. E. El-Taher, P. Harper, D. V. Churkin, S. I. Kablukov, E. V. Podivilov, and S. K. Turitsyn "Ultra-long Raman laser with a feedback based on the Rayleigh scattering" in Proceedings to the Conference on Lasers and Electro-Optics Europe, paper 5194622 (IEEE, Munich, 2009).
[9] S. K. Turitsyn, S. A. Babin, A. E. El-Taher, P. Harper, D. V. Churkin, S. I. Kablukov, J. D. Ania Castanon, V. Karalekas, and E. V. Podivilov "Random distributed feedback fibre laser" Nat. Photonics 4, 231-235 (2010).

[10] S. A. Babin, D. V. Churkin, A. E. Ismagulov, S. I. Kablukov, and E. V. Podivilov "Turbulence-induced square-root broadening of the Raman fiber laser output spectrum" 0pt. Lett. 33, 633-635 (2008).

[11] S. A. Babin, V. Karalekas, E. V. Podivilov V. K. Mezentsev, P. Harper, J. D. Ania Castanon, and S. K. Turitsyn "Turbulent broadening of optical spectra in ultralong Raman fiber lasers" Phys. Rev. A 77, 033803 (2008). 